\title{
Bayesian Estimation of Weighted Inverse Maxwell Distribution under Different Loss Functions
}

\author{
Aijaz Ahmad $^{1, *}$ and Rajnee Tripathi ${ }^{2}$ \\ ${ }^{1}$ Research Scholar, Department of Mathematics, Bhagwant University, Ajmer, India \\ e-mail: ahmadaijaz@gmail.com \\ ${ }^{2}$ Assistant Professor, Department of Mathematics, Bhagwant University, Ajmer, India
}

\begin{abstract}
In this study, the shape parameter of the weighted Inverse Maxwell distribution is estimated by employing Bayesian techniques. To produce posterior distributions, the extended Jeffery's prior and the Erlang prior are utilised. The estimators are derived from the squared error loss function, the entropy loss function, the precautionary loss function, and the Linex loss function. Furthermore, an actual data set is studied to assess the effectiveness of various estimators under distinct loss functions.
\end{abstract}

\section{Introduction}

The Maxwell-Boltzmann distribution, named after J.C. Maxwell and L. Boltzmann, is a continuous probability distribution that underpins the kinetic energy of gases as well as their indispensable characteristics such as pressure and diffusion. This distribution is also known as the distribution of velocities, energy and magnitude of momenta of molecules.

The probability density function (pdf) of Maxwell distribution is stated as

$$
f(x ; \theta)=\frac{4}{\sqrt{\pi}} \theta^{\frac{3}{2}} x^{2} e^{-\theta x^{2}} ; \quad x>0, \theta>0 .
$$

The related cumulative density function (cdf) is stated as

Received: November 26, 2021; Accepted: December 14, 2021

2020 Mathematics Subject Classification: 60-XX, 62-XX, 11-Kxx.

Keywords and phrases: Bayesian techniques, Jeffery's prior, Erlang prior, maximum likelihood estimation, loss functions. 


$$
F(x ; \theta)=\frac{2}{\sqrt{\pi}} \Gamma\left(\frac{3}{2}, \theta x^{2}\right) ; x>0, \theta>0 .
$$

Tyagi and Bhattacharya [8] studied this distribution for the first time as a lifetime model and addressed the Bayes and minimum variance unbiased estimation techniques for its parameters and reliability function.

Let $X$ denotes a random variable from Maxwell distribution, then the transformation $X=\frac{1}{Y}$ is said to follow inverse of Maxwell distribution having probability function (pdf) given by

$$
f(y ; \theta)=\frac{4}{\sqrt{\pi}} \theta^{\frac{3}{2}} \frac{1}{y^{4}} e^{-\frac{\theta}{y^{2}}} ; \quad y>0, \theta>0
$$

where $\theta$ represents the scale parameter.

The associated cumulative distribution function (cdf) of (1.3) is given by

$$
F(y ; \theta)=\frac{2 \theta}{\sqrt{\pi}} \Gamma\left(\frac{3}{2}, \frac{\theta}{y^{2}}\right) ; \quad y>0, \theta>0
$$

where $\Gamma(s, y)=\int_{y}^{\infty} t^{s-1} e^{-t} d t$ denotes an upper incomplete gamma function.

Recently, Ahmad et al. [3], studied the weighted analogue of inverse Maxwell distribution. With following probability density function

$$
f_{w}(y, \theta, k)=\frac{2 \theta^{\frac{3-k}{2}}}{\Gamma\left(\frac{3-k}{2}\right)} y^{k-4} e^{-\frac{\theta}{y^{2}}} ; \quad y>0, \theta, k>0 .
$$

The related cumulative distribution function is given as

$$
F_{w}(y, \theta, k)=\frac{\Gamma\left(\frac{3-k}{2}, \frac{\theta}{y^{2}}\right)}{\Gamma\left(\frac{3-k}{2}\right)} ; y>0, \theta, k>0
$$

where $\Gamma(s, y)=\int_{y}^{\infty} t^{s-1} e^{-t} d t$ denotes an upper incomplete gamma function.

Figures (1.1) and (1.2) represent some layouts of weighted inverse Maxwell distribution for distinct values of parameters. 


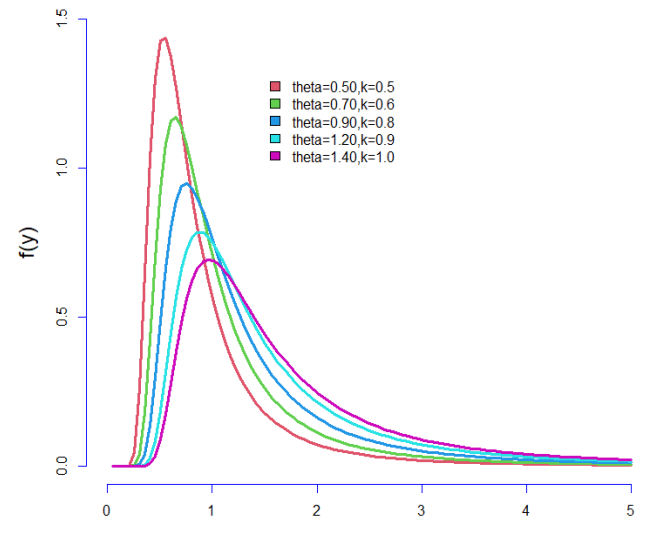

Fig 1.1 pdf of WIMD under different values of parameters

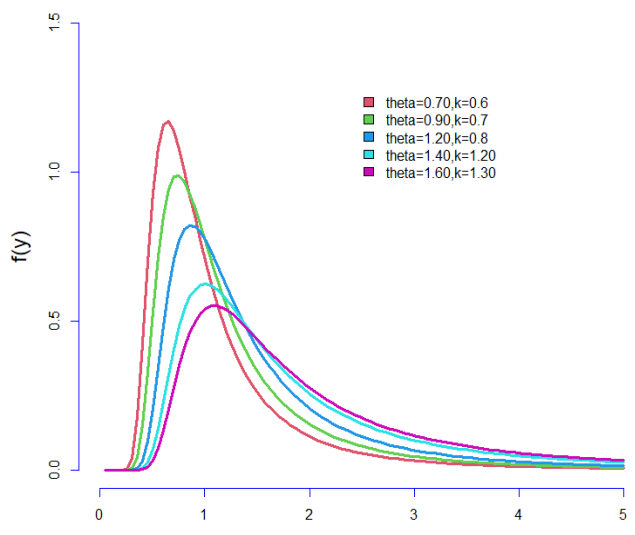

Fig 1.2 pdf of WIMD under different values of parameters

\section{Method of Maximum Likelihood Estimation of WIMD}

The estimation of parameters of weighted inverse Maxwell distribution is obtained by using the method of maximum likelihood estimation. Suppose $Y_{1}, Y_{2}, \ldots, Y_{n}$ be random samples of size $n$ from weighted inverse Maxwell distribution. Then the likelihood function of weighted inverse Maxwell distribution is given as

$$
l=\prod_{i=1}^{n} f_{w}\left(y_{i}, \theta, k\right)=\prod_{i=1}^{n} \frac{2 \theta^{\frac{3-k}{2}}}{\Gamma\left(\frac{3-k}{2}\right)} y_{i}^{k-4} e^{-\frac{\theta}{y_{i}{ }^{2}}} .
$$

The $\log$ likelihood function of (2.1) is given as

$$
\begin{aligned}
\ln l & =\ln \left(\frac{2 \theta^{\frac{3-k}{2}}}{\Gamma\left(\frac{3-k}{2}\right)}\right)^{n}+\sum_{i=1}^{n} \ln \left(y_{i}\right)^{k-4}-\sum_{i=1}^{n} \frac{\theta}{y_{i}^{2}} \\
& =2 \ln 2+n\left(\theta^{\frac{3-k}{2}}\right) \ln \theta-n \ln \Gamma\left(\frac{3-k}{2}\right)+(k-4) \sum_{i=1}^{n} \ln \left(y_{i}\right)-\sum_{i=1}^{n} \frac{\theta}{y_{i}^{2}} .
\end{aligned}
$$

Differentiate w.r.t. $\theta$, we get

$$
\frac{\partial \ln l}{\partial \theta}=\frac{n(3-k)}{2 \theta}-\sum_{i=1}^{n} \frac{1}{y_{i}^{2}} .
$$

Now equating (2.2), to zero, we get 


$$
\begin{gathered}
\Rightarrow \frac{n(3-k)}{2 \theta}-\sum_{i=1}^{n} \frac{1}{y_{i}^{2}}=0 \\
\hat{\theta}=\frac{n(3-k)}{2 \sum_{i=1}^{n} y_{i}^{-2}} .
\end{gathered}
$$

\section{Bayesian Estimation of WIMD}

In recent years, the Bayesian estimate technique has gained considerable attention for evaluating failure time data, and it has generally been presented as a substitute to the conventional techniques. By taking prior information into account, this formula accounts for the life-time distribution's subsequent (posterior) distribution. From a Bayesian perspective, picking prior(s) cannot be constrained by assuming that one's prior(s) is more suited than others. When there is minimal interpretive information about an unknown parameter, a non-informative prior is desirable. However, if one is familiar with the parameter (s), it is better to utilise informative prior. Ahmad et al. [1], investigated the estimate of parameters of a two-parameter Exponentiated gamma distribution in this way. Mudasir and Ahmad [6], studied the Bayesian estimation of weighted Erlang distribution. Raqab and Madi [7], studied Bayesian estimation for Exponentiated Rayleigh distribution. Kazmi et al. [9], studied Bayesian estimation for two component mixture of Maxwell distribution. Reshi et al. [10], studied Bayesian estimation of size biased classical gamma distribution. Muzamil et al. [11], studied Bayesian analysis of weighted Boltzmann-Maxwell distribution. Ahmad et al. [2], studied Bayesian estimation of inverse Ailamujia distribution used different loss functions. Ahmad et al. [4], investigated Bayesian estimation of the inverse Topp-Leone distribution assuming distinct loss functions. In this paper, we obtain Bayesian estimation of parameter $\hat{\theta}$ of weighted inverse Maxwell distribution under the assumption of extended Jeffrey's prior and Erlang distribution.

\subsection{Posterior distribution of WIMD under the assumption of extended Jeffrey's prior}

Suppose $y=\left(y_{1}, y_{2}, \ldots, y_{n}\right)$ denotes the $n$ recorded values of (1.5). Then its 
likelihood function is given by

$$
L(y \mid \theta)=\left(\frac{2}{\Gamma\left(\frac{3-k}{2}\right)}\right)^{n} \prod_{i=1}^{n} y_{i}^{k-4} \theta^{\frac{3 n-n k}{2}} e^{-\theta \sum_{i=1}^{n} y_{i}^{-2}} .
$$

We assume the prior distribution of $\theta$ to be extended Jeffery's prior proposed by AlKutubi [5], is given by

$$
g(\theta) \propto[I(\theta)]^{c}, \quad c \in R^{+}
$$

where $[I(\theta)]=-n E\left[\frac{\partial^{2} \log f(y, \theta)}{\partial^{2} \theta}\right]$ is the Fisher information matrix, for the distribution $(1.5)$

$$
g(\theta)=K\left[\frac{n}{\theta^{2}}\right]^{c}
$$

The posterior distribution of $\theta$ under the assumption of extended Jeffrey's prior, i.e., $g(\theta) \propto \frac{1}{\theta^{2 c}}$ is given by

$$
\begin{gathered}
h(\theta \mid y) \propto L(y \mid \theta) g(\theta) \\
h(\theta \mid y) \propto\left(\frac{2}{\Gamma\left(\frac{3-k}{2}\right)}\right)^{n} \prod_{i=1}^{n} y_{i}^{k-4} \theta^{\frac{3 n-n k}{2}} e^{-\theta \sum_{i=1}^{n} y_{i}^{-2}} \frac{1}{\theta^{2 c}} \\
h(\theta \mid y)=k \theta^{\frac{3 n-n k-4 c}{2}} e^{-\theta \sum_{i=1}^{n} y_{i}^{-2}}
\end{gathered}
$$

where $K$ is independent of $\theta$

$$
\begin{gathered}
K^{-1}=\int_{0}^{\infty} \theta^{\frac{3 n-n k-4 c}{2}} e^{-\theta \sum_{i=1}^{n} y_{i}^{-2}} d \theta \\
K^{-1}=\frac{\Gamma\left(\frac{3 n-n k-4 c+2}{2}\right)}{\left(\sum_{i=1}^{n} y_{i}^{-2}\right)^{\frac{3 n-n k-4 c+2}{2}}}
\end{gathered}
$$

Therefore

$$
K=\frac{\left(\sum_{i=1}^{n} y_{i}^{-2}\right)^{\frac{3 n-n k-4 c+2}{2}}}{\Gamma\left(\frac{3 n-n k-4 c+2}{2}\right)} .
$$


Hence the posterior distribution of $\theta$ is given by

$$
h(\theta \mid y)=\frac{T^{\frac{3 n-n k-4 c+2}{2}}}{\Gamma\left(\frac{3 n-n k-4 c+2}{2}\right)} \theta^{\frac{3 n-n k-4 c}{2}} e^{-\theta T}
$$

where $T=\sum_{i=1}^{n} y_{i}^{-2}$.

\subsection{Estimation under square error loss function}

The squared error loss function is defined as $l(\hat{\theta}, \theta)=c_{1}(\hat{\theta}-\theta)^{2}$ for some constant $c_{1}$. The risk function is given by

$$
\begin{aligned}
& R(\hat{\theta}, \theta)=E[l(\hat{\theta}, \theta)] \\
& =\int_{0}^{\infty} l(\hat{\theta}, \theta) h(\theta \mid y) d \theta \\
& =\int_{0}^{\infty} c_{1}(\hat{\theta}-\theta)^{2} \frac{T^{\frac{3 n-n k-4 c+2}{2}}}{\Gamma\left(\frac{3 n-n k-4 c+2}{2}\right)} \theta^{\frac{3 n-n k-4 c}{2}} e^{-\theta T} d \theta \\
& =c_{1} \frac{T^{\frac{3 n-n k-4 c+2}{2}}}{\Gamma\left(\frac{3 n-n k-4 c+2}{2}\right)} \int_{0}^{\infty}(\hat{\theta}-\theta)^{2} \theta^{\frac{3 n-n k-4 c}{2}} e^{-\theta T} d \theta \\
& =c_{1} \frac{T^{\frac{3 n-n k-4 c+2}{2}}}{\Gamma\left(\frac{3 n-n k-4 c+2}{2}\right)}\left[\begin{array}{l}
\hat{\theta}^{2} \int_{0}^{\infty} \theta^{\frac{3 n-n k-4 c}{2}} e^{-\theta T} d \theta+\int_{0}^{\infty} \theta^{\frac{3 n-n k-4 c+4}{2}} e^{-\theta T} d \theta \\
-2 \hat{\theta} \int_{0}^{\infty} \theta^{\frac{3 n-n k-4 c+2}{2}} e^{-\theta T} d \theta
\end{array}\right] \\
& =c_{1} \frac{T^{\frac{3 n-n k-4 c+2}{2}}}{\Gamma\left(\frac{3 n-n k-4 c+2}{2}\right)}\left[\begin{array}{l}
\hat{\theta}^{2} \frac{\Gamma\left(\frac{3 n-n k-4 c+2}{2}\right)}{T^{\frac{3 n-n k-4 c+2}{2}}}+\frac{\Gamma\left(\frac{3 n-n k-4 c+6}{2}\right)}{T^{\frac{3 n-n k-4 c+6}{2}}} \\
-2 \hat{\theta} \frac{\Gamma\left(\frac{3 n-n k-4 c+4}{2}\right)}{T^{\frac{3 n-n k-4 c+4}{2}}}
\end{array}\right] \\
& =c_{1}\left[\hat{\theta}^{2}+\frac{(3 n-n k-4 c+2)(3 n-n k-4 c+4)}{4 T^{2}}-\hat{\theta} \frac{(3 n-n k-4 c+2)}{T}\right] \text {. }
\end{aligned}
$$

Now solve $\frac{\partial R(\widehat{\theta}, \theta)}{\partial \widehat{\theta}}=0$, we get 


$$
\hat{\theta}_{s}=\frac{(3 n-n k-4 c+2)}{2 T} .
$$

\subsection{Estimation under entropy loss function}

The entropy loss function is defined as $l(\delta)=b[\delta-\log (\delta)-1] ; b>0, \delta=\frac{\widehat{\theta}}{\theta}$. The risk function is given by

$$
\begin{aligned}
& R(\hat{\theta}, \theta)=E[l(\delta)] \\
= & \int_{0}^{\infty} l(\delta) h(\theta \mid y) d \theta \\
= & \int_{0}^{\infty} b[\delta-\log (\delta)-1] h(\theta \mid y) d \theta \\
= & b \frac{T^{\frac{3 n-n k-4 c+2}{2}}}{\Gamma\left(\frac{3 n-n k-4 c+2}{2}\right)} \int_{0}^{\infty}\left[\frac{\hat{\theta}}{\theta}-\log \left(\frac{\hat{\theta}}{\theta}\right)-1\right] \theta^{\frac{3 n-n k-4 c}{2}} e^{-\theta T} d \theta \\
= & b \frac{T^{\frac{3 n-n k-4 c+2}{2}}}{\Gamma\left(\frac{3 n-n k-4 c+2}{2}\right)}\left[\begin{array}{l}
\theta \\
0
\end{array} \int_{0}^{\infty} \theta^{\frac{3 n-n k-4 c-2}{2}} e^{-\theta T} d \theta-\log (\theta) \theta^{\frac{3 n-n k-4 c}{2}} e^{-\theta T} d \theta-\int_{0}^{\infty} \theta^{\frac{3 n-n k-4 c}{2}} e^{-\theta T} d \theta\right] \\
= & b\left[\frac{2 \hat{\theta} T}{3 n-n k-4 c}-\log (\hat{\theta})+\frac{\left.\Gamma^{\frac{3 n-n k-4 c}{2}} e^{-\theta T} d \theta\right]}{\Gamma\left(\frac{3 n-n k-4 c}{2}\right)} \int^{\frac{3 n-n k-4 c}{2}}-1\right] \\
= & b\left[\frac{2 \hat{\theta} T}{3 n-n k-4 c}-\log (\hat{\theta})+\psi^{\prime}\left(\frac{3 n-n k-4 c}{2}\right)-1\right]
\end{aligned}
$$

where $\psi^{\prime}($.$) denotes the digamma function$

Now solve $\frac{\partial R(\widehat{\theta}, \theta)}{\partial \widehat{\theta}}=0$, we get

$$
\hat{\theta}_{e}=\frac{(3 n-3 k-4 c)}{2 T} .
$$

\subsection{Estimation under precautionary loss function}

The entropy loss function is defined as $l(\hat{\theta}, \theta)=\frac{(\widehat{\theta}-\theta)^{2}}{\widehat{\theta}}$. The risk function is given by 


$$
\begin{aligned}
& R(\hat{\theta}, \theta)=E[l(\hat{\theta}, \theta)] \\
= & \int_{0}^{\infty} l(\hat{\theta}, \theta) h(\theta \mid y) d \theta \\
= & \frac{T^{\frac{3 n-n k-4 c+2}{2}}}{\Gamma\left(\frac{3 n-n k-4 c+2}{2}\right)} \int_{0}^{\infty} \frac{(\hat{\theta}-\theta)^{2}}{\hat{\theta}} \theta^{\frac{3 n-n k-4 c}{2}} e^{-\theta T} d \theta \\
= & \frac{T^{\frac{3 n-n k-4 c+2}{2}}}{\Gamma\left(\frac{3 n-n k-4 c+2}{2}\right)}\left[-2 \int_{0}^{\infty} \theta^{\frac{3 n-n k-4 c}{2}} e^{-\theta T} d \theta+\frac{1}{\hat{\theta}} \int_{0}^{\infty} \theta^{\frac{3 n-n k-n k-4 c+4}{2}} e^{-\theta T} d \theta\right] \\
= & {\left[\hat{\theta}+\frac{(3 n-n k-\theta T}{\theta^{\frac{3 n-2}{2}}} e^{-4 c+2)(3 n-n k-4 c+4)}\right.} \\
4 \hat{\theta} T^{2} & \left.-\frac{(3 n-n k-4 c+2)}{T}\right] .
\end{aligned}
$$

Now solve $\frac{\partial R(\widehat{\theta}, \theta)}{\partial \widehat{\theta}}=0$, we get

$$
\hat{\theta}_{p}=\frac{\sqrt{(3 n-3 k-4 c+2)(3 n-3 k-4 c+4)}}{2 T} .
$$

\subsection{Estimation under Linex loss function}

The Linex loss function is defined as $l(\hat{\theta}, \theta)=\exp \left\{b_{1}(\hat{\theta}-\theta)\right\}-b_{1}(\hat{\theta}-\theta)-1$. The risk function is given by

$$
\begin{aligned}
& R(\hat{\theta}, \theta)=E[l(\hat{\theta}, \theta)] \\
&= \int_{0}^{\infty} l(\hat{\theta}, \theta) h(\theta \mid y) d \theta \\
&= \frac{T^{\frac{3 n-n k-4 c+2}{2}}}{\Gamma\left(\frac{3 n-n k-4 c+2}{2}\right)} \int_{0}^{\infty}\left[\exp \left\{b_{1}(\hat{\theta}-\theta)\right\}-b_{1}(\hat{\theta}-\theta)-1\right] \theta^{\frac{3 n-n k-4 c+2}{2}} e^{-\theta T} d \theta \\
&= \frac{T^{\frac{3 n-n k-4 c+2}{2}}}{\Gamma\left(\frac{3 n-n k-4 c+2}{2}\right)}\left[e^{b_{1} \widehat{\theta}_{1}} \int_{0}^{\infty} \theta^{\frac{3 n-n k-4 c}{2}} e^{-\theta\left(b_{1}+T\right)} d \theta-b_{1} \hat{\theta} \int_{0}^{\infty} \theta^{\frac{3 n-n k-4 c}{2}} e^{-\theta T} d \theta\right] \\
&+b_{1} \int_{0}^{\infty} \theta^{\frac{3 n-n k-4 c+2}{2}} e^{-\theta T} d \theta-\int_{0}^{\infty} \theta^{\frac{3 n-n k-4 c}{2}} e^{-\theta T} d \theta
\end{aligned}
$$


$=\left[e^{b_{1} \widehat{\theta}}\left(\frac{T}{b_{1}+T}\right)^{\frac{3 n-n k-4 c+2}{2}}-b_{1} \hat{\theta}+b_{1} \frac{(3 n-n k-4 c+2)}{2 T}-1\right]$.

Now solve $\frac{\partial R(\widehat{\theta}, \theta)}{\partial \widehat{\theta}}=0$, we get

$$
\hat{\theta}_{l}=\frac{(3 n-n k-4 c+2)}{2 b_{1}} \log \left(\frac{b_{1}+T}{T}\right) .
$$

\subsection{Posterior distribution of WIMD under the assumption of Erlang prior}

Suppose $y=\left(y_{1}, y_{2}, \ldots, y_{n}\right)$ denotes the $n$ recorded values of (1.5). Then its likelihood function is given by

$$
L(y \mid \theta)=\left(\frac{2}{\Gamma\left(\frac{3-k}{2}\right)}\right)^{n} \prod_{i=1}^{n} y_{i}^{k-4} \theta^{\frac{3 n-n k}{2}} e^{-\theta \sum_{i=1}^{n} y_{i}^{-2}} .
$$

We assume the prior distribution of $\theta$ to be Erlang prior.

The posterior distribution of $\theta$ under the assumption of Erlang prior i.e. $g(\theta) \propto$ $\frac{a^{b}}{\Gamma(b)} \theta^{b-1} e^{-a \theta}$ is given by

$$
\begin{gathered}
h(\theta \mid y) \propto L(y \mid \theta) g(\theta) \\
h(\theta \mid y) \propto\left(\frac{2}{\Gamma\left(\frac{3-k}{2}\right)}\right)^{n} \prod_{i=1}^{n} y_{i}^{k-4} \theta^{\frac{3 n-n k}{2}} e^{-\theta \sum_{i=1}^{n} y_{i}^{-2}} \frac{a^{b}}{\Gamma(b)} \theta^{b-1} e^{-a \theta} \\
h(\theta \mid y)=k \theta^{\frac{3 n-n k+2 b}{2}-1} e^{-\theta\left(a+\sum_{i=1}^{n} y_{i}^{-2}\right)}
\end{gathered}
$$

where $K$ is independent of $\theta$

$$
\begin{gathered}
K^{-1}=\int_{0}^{\infty} \theta^{\frac{3 n-n k+2 b}{2}-1} e^{-\theta\left(a+\sum_{i=1}^{n} y_{i}^{-2}\right)} d \theta \\
K^{-1}=\frac{\Gamma\left(\frac{3 n-n k+2 b}{2}\right)}{\left(a+\sum_{i=1}^{n} y_{i}^{-2}\right)^{\frac{3 n-n k+2 b}{2}}} .
\end{gathered}
$$


Therefore

$$
K=\frac{\left(a+\sum_{i=1}^{n} y_{i}^{-2}\right)^{\frac{3 n-n k+2 b}{2}}}{\Gamma\left(\frac{3 n-n k+2 b}{2}\right)} .
$$

Hence the posterior distribution of $\theta$ is given by

$$
h(\theta \mid y)=\frac{(a+T)^{\frac{3 n-n k+2 b}{2}}}{\Gamma\left(\frac{3 n-n k+2 b}{2}\right)} \theta^{\frac{3 n-n k+2 b}{2}-1} e^{-\theta(a+T)}
$$

where $T=\sum_{i=1}^{n} y_{i}^{-2}$.

\subsection{Estimation under square error loss function}

The squared error loss function is defined as $l(\hat{\theta}, \theta)=c_{1}(\hat{\theta}-\theta)^{2}$ for some constant $c_{1}$. The risk function is given by

$$
\begin{aligned}
& R(\hat{\theta}, \theta)=E[l(\hat{\theta}, \theta)] \\
&= \int_{0}^{\infty} l(\hat{\theta}, \theta) h(\theta \mid y) d \theta \\
&= c_{1} \frac{(a+T)^{\frac{3 n-n k+2 b}{2}}}{\Gamma\left(\frac{3 n-n k+2 b}{2}\right)} \int_{0}^{\infty}(\hat{\theta}-\theta)^{2} \theta^{\frac{3 n-n k+2 b}{2}-1} e^{-\theta(a+T)} d \theta \\
&= c_{1} \frac{(a+T)^{\frac{3 n-n k+2 b}{2}}}{\Gamma\left(\frac{3 n-n k+2 b}{2}\right)}\left[-2 \hat{\theta} \int_{0}^{\infty} \int_{0}^{\infty} \theta^{\frac{3 n-n k+2 b}{2}-1} e^{-\theta(a+T)} d \theta+\int_{0}^{\infty} \theta^{\frac{3 n-n k+2 b+4}{2}-1} e^{-\theta(a+T)} d \theta\right] \\
&= c_{1}\left[\hat{\theta}^{2}+\frac{(3 n-n k+2}{2} e^{-\theta(a+T)} d \theta\right. \\
& 4(a+T)^{2}
\end{aligned}
$$

Now solve $\frac{\partial R(\widehat{\theta}, \theta)}{\partial \widehat{\theta}}=0$, we get

$$
\hat{\theta}_{s}=\frac{(3 n-n k+2 b)}{2(a+T)} .
$$




\subsection{Estimation under entropy loss function}

The entropy loss function is defined as $l(\delta)=b[\delta-\log (\delta)-1] ; b>0, \delta=\frac{\widehat{\theta}}{\theta}$. The risk function is given by

$$
\begin{aligned}
& R(\hat{\theta}, \theta)=E[l(\delta)] \\
& =\int_{0}^{\infty} l(\delta) h(\theta \mid y) d \theta \\
& =\int_{0}^{\infty} b_{1}[\delta-\log (\delta)-1] h(\theta \mid y) d \theta \\
& =b_{1} \frac{(a+T)^{\frac{3 n-n k+2 b}{2}}}{\Gamma\left(\frac{3 n-n k+2 b}{2}\right)} \int_{0}^{\infty}\left[\frac{\hat{\theta}}{\theta}-\log \left(\frac{\hat{\theta}}{\theta}\right)-1\right] \theta^{\frac{3 n-n k+2 b}{2}-1} e^{-\theta(a+T)} d \theta \\
& \left.=b_{1} \frac{(a+T)^{\frac{3 n-n k+2 b}{2}}}{\Gamma\left(\frac{3 n-n k+2 b}{2}\right)}\left[\hat{\theta} \int_{0}^{\infty} \theta^{\frac{3 n-n k+2 b-2}{2}-1} e^{-\theta(a+T)} d \theta-\log (\hat{\theta}) \int_{0}^{\infty} \theta^{\frac{3 n-n k+2 b}{2}-1} e^{-\theta(a+T)} d \theta\right]+\int_{0}^{\infty} \log (\theta) \theta^{\frac{3 n-n k+2 b}{2}-1} e^{-\theta(a+T)} d \theta-\int_{0}^{\infty} \theta^{\frac{3 n-n k+2 b}{2}-1} e^{-\theta(a+T)} d \theta\right] \\
& =b_{1}\left[\frac{2 \hat{\theta}(a+T)}{(3 n-n k+2 b-2)}-\log (\hat{\theta})+\psi^{\prime}\left(\frac{3 n-n k+2 b}{2}\right)-1\right] \text {. }
\end{aligned}
$$

Now solve $\frac{\partial R(\widehat{\theta}, \theta)}{\partial \widehat{\theta}}=0$, we get

$$
\hat{\theta}_{e}=\frac{(3 n-n k+2 b-2)}{2(a+T)} .
$$

\subsection{Estimation under precautionary loss function}

The entropy loss function is defined as $l(\hat{\theta}, \theta)=\frac{(\widehat{\theta}-\theta)^{2}}{\widehat{\theta}}$. The risk function is given by

$$
\begin{aligned}
& R(\hat{\theta}, \theta)=E[l(\hat{\theta}, \theta)] \\
= & \int_{0}^{\infty} l(\hat{\theta}, \theta) h(\theta \mid y) d \theta \\
= & \frac{(a+T)^{\frac{3 n-n k+2 b}{2}}}{\Gamma\left(\frac{3 n-n k+2 b}{2}\right)} \int_{0}^{\infty}\left(\hat{\theta}+\frac{\theta^{2}}{\hat{\theta}}-2 \theta\right) \theta^{\frac{3 n-n k+2 b}{2}-1} e^{-\theta(a+T)} d \theta
\end{aligned}
$$


$=\frac{(a+T)^{\frac{3 n-n k+2 b}{2}}}{\Gamma\left(\frac{3 n-n k+2 b}{2}\right)}\left[\begin{array}{l}\left.\hat{\theta} \int_{0}^{\infty} \theta^{\frac{3 n-n k+2 b}{2}-1} e^{-\theta(a+T)} d \theta+\frac{1}{\hat{\theta}} \int_{0}^{\infty} \theta^{\frac{3 n-n k+2 b}{2}+1} e^{-\theta(a+T)} d \theta\right] \\ -2 \int_{0}^{\infty} \theta^{\frac{3 n-n k+2 b}{2}} e^{-\theta(a+T)} d \theta\end{array}\right]$

$=\left[\hat{\theta}+\frac{(3 n-n k+2 b)(3 n-n k+2 b+2)}{4 \hat{\theta}(a+T)^{2}}-\frac{(3 n-n k+2 b)}{(a+T)}\right]$.

Now solve $\frac{\partial R(\widehat{\theta}, \theta)}{\partial \widehat{\theta}}=0$, we get

$$
\hat{\theta}_{e}=\frac{\sqrt{(3 n-n k+2 b)(3 n-n k+2 b+2)}}{2(a+T)} .
$$

\subsection{Estimation under Linex loss function}

The Linex loss function is defined as $l(\hat{\theta}, \theta)=\exp \left\{b_{1}(\hat{\theta}-\theta)\right\}-b_{1}(\hat{\theta}-\theta)-1$. The risk function is given by

$$
\begin{aligned}
& R(\hat{\theta}, \theta)=E[l(\hat{\theta}, \theta)] \\
= & \int_{0}^{\infty} l(\hat{\theta}, \theta) h(\theta \mid y) d \theta \\
= & \frac{(a+T)^{\frac{3 n-n k+2 b}{2}}}{\Gamma\left(\frac{3 n-n k+2 b}{2}\right)} \int_{0}^{\infty}\left[\exp \left\{b_{1}(\hat{\theta}-\theta)\right\}-b_{1}(\hat{\theta}-\theta)-1\right] \theta^{\frac{3 n-n k+2 b}{2}} e^{-\theta(a+T)} d \theta \\
= & \frac{T^{\frac{3 n-n k+2 b}{2}}}{\Gamma\left(\frac{3 n-n k+2 b}{2}\right)}\left[e^{b_{1} \widehat{\theta}_{1}} \int_{0}^{\infty} \theta^{\frac{3 n-n k+2 b}{2}-1} e^{-\theta\left(b_{1}+a+T\right)} d \theta-b_{1} \hat{\theta} \int_{0}^{\infty} \theta_{0}^{\frac{3 n-n k+2 b}{2}-1} e^{-\theta(a+T)} d \theta-\int_{0}^{\infty} \theta^{\frac{3 n-n k+2 b}{2}-1} e^{-\theta(a+T)} d \theta\right. \\
= & {\left[e^{b_{1} \hat{\theta}\left(\frac{a+T)}{2}\left(\frac{a+T}{b_{1}+a+T}\right)\right.}\right] }
\end{aligned}
$$

Now solve $\frac{\partial R(\widehat{\theta}, \theta)}{\partial \widehat{\theta}}=0$, we get

$$
\hat{\theta}_{l}=\frac{(3 n-n k+2 b)}{2 b_{1}} \log \left(\frac{b_{1}+a+T}{a+T}\right) .
$$




\section{Application}

This section provides an application that assesses the efficacy of estimators and the posterior risk of various loss functions. The following is the data set.

Data:-The data represents a COVID-19 data set from Canada that spans 36 days, from 10 April to 15 May 2020, as shown in the URL (https://covid19.who.int/). These data is based on the famine fatality rate. The information is as follows:

$0.2240,0.2189,0.2105,0.2266,0.0987,0.1147,0.3353,0.2563,0.2466,0.2847,0.2150$, 0.1821, 0.1200, 0.4206, 0.3456, 0.3045, 0.2903, 0.3377, 0.1639, 0.1350, 0.3866, 0.4678, $0.3515,0.3232$

The Bayes estimates and posterior risks of the posterior distribution employing different loss functions are as below, with the posterior risk in parentheses

$\hat{\theta}_{s}=$ Square error loss function, $\quad \hat{\theta}_{e}=$ Estimation under Entropy,

$\hat{\theta}_{p}=$ Estimation under Precautionary, $\hat{\theta}_{l}=$ Estimation under LINEX

Table 4.1: Bayes estimation and posterior risks using Jeffery's prior.

\begin{tabular}{|c|c|c|c|c|c|c|c|}
\hline$\hat{\theta}$ & $\hat{k}$ & $C$ & $\hat{\theta}_{s}$ & $\theta_{e}$ & $\theta_{p}$ & \multicolumn{2}{|c|}{$\hat{\theta}_{l}$} \\
\cline { 5 - 8 } & & & & & & $b_{1}=0.1$ & $b_{1}=0.5$ \\
\hline 1.0 & 0.5 & 0.5 & 0.0498 & 0.0481 & 0.0506 & 0.0004 & 0.0124 \\
& & & $(8.2 \mathrm{e}-05)$ & $(6.383)$ & $(2.385)$ & $(4.1 \mathrm{e}-07)$ & $(1.03 \mathrm{e}-05)$ \\
\cline { 3 - 7 } & & 1.0 & 0.0481 & 0.0465 & 0.0489 & 0.0004 & 0.0120 \\
& & & $(7.9 \mathrm{e}-05)$ & $(6.382)$ & $(2.307)$ & $(3.9 \mathrm{e}-07)$ & $(9.9 \mathrm{e}-06)$ \\
\cline { 3 - 7 } & & 1.5 & 0.0465 & 0.0448 & 0.0473 & 0.0004 & 0.0120 \\
& & & $(7.7 \mathrm{e}-05)$ & $(6.381)$ & $(2.229)$ & $(3.8 \mathrm{e}-07)$ & $(9.6 \mathrm{e}-06)$ \\
\hline 2.0 & \multirow{2}{*}{1.5} & 0.5 & 0.0298 & 0.0282 & 0.0307 & 0.0002 & 0.0074 \\
& & & $(4.9 \mathrm{e}-05)$ & $(6.370)$ & $(1.447)$ & $(2.4 \mathrm{e}-07)$ & $(6.2 \mathrm{e}-06)$ \\
\cline { 3 - 7 } & & 1.0 & 0.0282 & 0.0265 & 0.0290 & 0.0002 & 0.0070 \\
& & & $(4.6 \mathrm{e}-05)$ & $(6.368)$ & $(1.369)$ & $(2.3 \mathrm{e}-07)$ & $(5.8 \mathrm{e}-06)$ \\
\cline { 3 - 7 } & & 1.5 & 0.0265 & 0.0249 & 0.0273 & 0.0002 & 0.0066 \\
& & & $(4.4 \mathrm{e}-07)$ & $(6.366)$ & $(1.291)$ & $(2.2-07)$ & $(5.5 \mathrm{e}-06)$ \\
\hline 33.0 & \multirow{2}{*}{2.0} & 0.5 & 0.0199 & 0.018 & 0.0207 & 0.0001 & 0.0049 \\
& & & $(3.3 \mathrm{e}-05)$ & $(6.354)$ & $(0.978)$ & $(1.6 \mathrm{e}-07)$ & $(4.1-06)$ \\
\cline { 3 - 7 } & & 1.0 & 0.0182 & 0.016 & 0.019 & 0.0001 & 0.0045 \\
\hline
\end{tabular}




\begin{tabular}{|c|c|c|c|c|c|c|}
\hline \multirow{3}{*}{} & & $(3.0 \mathrm{e}-05)$ & $(6.349)$ & $(0.899)$ & $(1.5 \mathrm{e}-07)$ & $(3.7-06)$ \\
\cline { 2 - 7 } & 1.5 & 0.0160 & 0.0149 & 0.0174 & 0.0001 & 0.0045 \\
& & $(2.7 \mathrm{e}-05)$ & $(6.343)$ & $(0.821)$ & $(1.3 \mathrm{e}-07)$ & $(3.4-06)$ \\
\hline
\end{tabular}

Table 4.2: Bayes estimation and posterior risks using Erlang prior.

\begin{tabular}{|c|c|c|c|c|c|c|c|c|}
\hline \multirow[t]{2}{*}{$\hat{\theta}$} & \multirow[t]{2}{*}{$\widehat{k}$} & \multirow[t]{2}{*}{$a$} & \multirow[t]{2}{*}{$b$} & \multirow[t]{2}{*}{$\hat{\theta}_{s}$} & \multirow[t]{2}{*}{$\theta_{e}$} & \multirow[t]{2}{*}{$\theta_{p}$} & \multicolumn{2}{|c|}{$\hat{\theta}_{l}$} \\
\hline & & & & & & & $b_{1}=0.1$ & $b_{1}=0.5$ \\
\hline \multirow[t]{3}{*}{1.0} & \multirow[t]{3}{*}{0.5} & 0.5 & 0.5 & $\begin{array}{c}0.0506 \\
(8.3 \mathrm{e}-05)\end{array}$ & $\begin{array}{l}8.1 \mathrm{e}-05 \\
(6.418)\end{array}$ & $\begin{array}{l}0.0514 \\
(2.424)\end{array}$ & $\begin{array}{c}0.0005 \\
(4 . e-07)\end{array}$ & $\begin{array}{c}0.0126 \\
(1 . e-07)\end{array}$ \\
\hline & & 1.0 & 0.7 & $\begin{array}{c}0.0509 \\
(8.4 \mathrm{e}-05)\end{array}$ & $\begin{array}{c}8.1 \mathrm{e}-05 \\
(6.418)\end{array}$ & $\begin{array}{l}0.0517 \\
(2.438)\end{array}$ & $\begin{array}{c}0.0005 \\
(4 . e-07)\end{array}$ & $\begin{array}{c}0.0127 \\
(1 . e-05)\end{array}$ \\
\hline & & 1.5 & 1.0 & $\begin{array}{c}0.0513 \\
(8.5 \mathrm{e}-05)\end{array}$ & $\begin{array}{l}8.2 \mathrm{e}-05 \\
(6.419)\end{array}$ & $\begin{array}{c}0.0521 \\
(2.461)\end{array}$ & $\begin{array}{c}0.0005 \\
(4 . e-07)\end{array}$ & $\begin{array}{c}0.0128 \\
(1 . e-05)\end{array}$ \\
\hline \multirow[t]{3}{*}{2.0} & \multirow[t]{3}{*}{1.5} & 0.5 & 0.5 & $\begin{array}{c}0.0306 \\
(8.0 \mathrm{e}-05)\end{array}$ & $\begin{array}{l}4.8 \mathrm{e}-05 \\
(6.429)\end{array}$ & $\begin{array}{l}0.0315 \\
(1.486)\end{array}$ & $\begin{array}{c}0.0003 \\
(2 . e-07)\end{array}$ & $\begin{array}{c}0.0076 \\
(6 . e-06)\end{array}$ \\
\hline & & 1.0 & 0.7 & $\begin{array}{c}0.0310 \\
(5.1 \mathrm{e}-05)\end{array}$ & $\begin{array}{l}4.8 \mathrm{e}-05 \\
(6.430)\end{array}$ & $\begin{array}{c}0.0318 \\
(1.501)\end{array}$ & $\begin{array}{c}0.0003 \\
(2 . e-07)\end{array}$ & $\begin{array}{c}0.0077 \\
(6 . e-06)\end{array}$ \\
\hline & & 1.5 & 1.0 & $\begin{array}{c}0.0314 \\
(5.2 \mathrm{e}-05)\end{array}$ & $\begin{array}{l}4.9 \mathrm{e}-05 \\
(6.430)\end{array}$ & $\begin{array}{l}0.0322 \\
(1.523)\end{array}$ & $\begin{array}{c}0.0003 \\
(2 . e-07)\end{array}$ & $\begin{array}{c}0.0078 \\
(6 . e-06)\end{array}$ \\
\hline \multirow[t]{3}{*}{3.0} & \multirow[t]{3}{*}{2.0} & 0.5 & 0.5 & $\begin{array}{c}0.0207 \\
(3.4 \mathrm{e}-05)\end{array}$ & $\begin{array}{l}3.1 \mathrm{e}-05 \\
(6.444)\end{array}$ & $\begin{array}{l}0.0215 \\
(1.016)\end{array}$ & $\begin{array}{c}0.0002 \\
(1 . e-07)\end{array}$ & $\begin{array}{c}0.0051 \\
(4 . e-07)\end{array}$ \\
\hline & & 1.0 & 0.7 & $\begin{array}{c}0.0210 \\
(3.4 \mathrm{e}-05)\end{array}$ & $\begin{array}{l}3.2 \mathrm{e}-05 \\
(6.444)\end{array}$ & $\begin{array}{l}0.0218 \\
(1.032)\end{array}$ & $\begin{array}{c}0.0002 \\
(1 . e-07)\end{array}$ & $\begin{array}{c}0.0052 \\
(4 . e-06)\end{array}$ \\
\hline & & 1.5 & 1.0 & $\begin{array}{c}0.0215 \\
(3.5 \mathrm{e}-05)\end{array}$ & $\begin{array}{l}3.2 \mathrm{e}-05 \\
(6.444)\end{array}$ & $\begin{array}{l}0.0223 \\
(1.055)\end{array}$ & $\begin{array}{c}0.0002 \\
(1 . e-07)\end{array}$ & $\begin{array}{c}0.0053 \\
(4 . e-06)\end{array}$ \\
\hline
\end{tabular}

Among other loss functions, it is evident from Table 4.1 and Table 4.2. That the Linex error loss function shows smaller Bayes posterior risk under the both assumptions (extended Jeffery's prior and Erlang prior). According to decision rule of less Bayes posterior risk, we accomplish that Linex error loss function is more useful than others.

\section{Conclusion}

In this research, we first achieved the Bayes posterior distribution and parameter estimate of the weighted inverse Maxwell distribution by utilising both informative and non-informative priors. We have examined several loss functions, with the Linex error loss function offering the least Bayes posterior risk. The effectiveness of the estimators was subsequently attained by an application. 


\section{References}

[1] A. Ahmad, S.P. Ahmad and A. Ahmed, Estimation of parameters of two parameter exponentiated gamma distribution using R software, Pakistan Journal of Statistics 34(5) (2018), 359-375.

[2] A. Ahmad, S.Q. Ul Ain, A. Ahmad and R. Tripathi, Bayesian estimation of inverse Ailamujia distribution using different loss functions, Journal of Xi'an University of Architecture and Technology XII(XI) (2020), 226-238.

[3] A. Ahmad, A. Ahmad and R. Tripathi, Weighted analogue of inverse Maxwell distribution with applications, International Journal of Statistics and Mathematics 7(1) (2020), 146-153.

[4] A. Ahmad, S.Q. Ul Ain, A. Ahmad and R. Tripathi, Bayesian analysis of inverse ToppLeone distribution using different loss functions, Journal of Xi'an University of Architecture and Technology XII(XII) (2020), 581-596.

[5] Al. Kutubi, On comparison estimation procedure for parameter and survival function, Iraqi Journal of Statistical Science 9 (2005), 1-14.

[6] S. Mudasir and S.P. Ahmad, Parameter estimation of weighted distribution using software, Mathematical Theory and Modelling 7(6) (2017), 1-21.

[7] M.Z. Raqab and M.T. Madi, Bayesian Analysis for the exponentiated Rayleigh distribution, METRON-International Journal of Statistics 67(3) (2009), 269-288.

[8] R.K. Tyagi and S.K. Bhattacharya, Bayes estimation of the Maxwell's velocity distribution function, Statistica 29(4) (1989), 563-567.

[9] S. Kazmi, M. Aslam and S. Ali, On the Bayesian estimation for two component mixture of Maxwell distribution, assuming type-1 censored data, International Journal of Applied Science and Technology 2(1) (2012), 197-218,

[10] J.A. Reshi, A. Ahmed and K.A. Mir, Bayesian estimation of size biased classical gamma distribution, Journal of Reliability and Statistical Studies 7(1) (2014), 31-42.

[11] J. Muzamil, J.A. Reshi and T. Rajnee, Bayesian analysis of weighted BoltzmannMaxwell distribution; a simulation study, Journal of Statistical Applications and Probability 10(3) (2021), 795-806. https://doi.org/10.18576/jsap/100317

This is an open access article distributed under the terms of the Creative Commons Attribution License (http://creativecommons.org/licenses/by/4.0/), which permits unrestricted, use, distribution and reproduction in any medium, or format for any purpose, even commercially provided the work is properly cited. 